\title{
miRNA-mRNA pairs are differently expressed in African American men
}

Comparison of mRNA and microRNA (miRNA) expression in prostate cancer samples from African American men and American men of European descent has shed new light on the possible cause of the disparity between these two populations with regards to this disease.

Using integrative genomics, Wang and colleagues identified 22 miRNAs that are specific to prostate cancer in African American men and 18 miRNAs specific to prostate cancer in European Americans. Moreover, comparison of miRNA expression between the two populations showed 10 miRNAs are significantly enriched or depleted in samples from African American men compared with European American men.

Further analysis identified 150 reciprocally expressed miRNA-mRNA pairings and 124 Kyoto Encyclopedia of Genes and Genomes (KEGG) pathways significantly associated with prostate cancer in African American men. These pathways included ERBB, MTOR, WNT, JAK-STAT, TGF- $\beta$, P53 and VEGF. Most notably, genes associated with the ERBB pathway were upregulated in prostate-cancer samples from African American men and downregulated in samples from European American men.

\section{4 ...10 miRNAs are significantly enriched or depleted... 77}

Mapping population-associated miRNA-mRNA pairings on to the ERBB signalling pathway revealed 17 specific, two enriched and four depleted African-American miRNAs predicted to target 56 of 85 ERBB signalling genes and also 14 novel miRNA-mRNA pairs. Dr Norman Lee, principle investigator, told Nature Reviews Urology"Disrupting miRNA-mRNA pairings resulted in a less tumorigenic phenotype in African American prostate cancer cell lines, with the opposite effect observed in European cancer cell lines, suggesting a molecular basis for prostate cancer disparities among different populations.” Dr Bi-Dar Wang, lead author, added "The expression profiles of miR-133a-MCL1, miR-513c-STAT1, miR-96-FOXO3A, miR-145-ITPR2 and $\mathrm{miR}-34 \mathrm{a}-P P P 2 R 2 A$ pairings may serve as indicators for evaluating prostate cancer aggressiveness in patients, especially in the African-American population", concluding, "A patient's ethnic background could be an important factor in future clinical studies assessing the efficacy of EGFR, PI3K, ATK and/or mTOR inhibitors."

\section{Louise Stone}

Original article Wang, B.-D. et al. Identification and functional validation of reciprocal microRNA-mRNA pairings in African American prostate cancer disparities. Clin. Cancer Res. doi:10.1158/1078-0432.CCR-14-1566 